\title{
Avaliação da satisfação de pacientes oncológicos com o serviço de saúde em um hospital público no Brasil
}

\section{Evaluation of oncology patients' satisfaction with health service in a public hospital in Brazil}

\author{
Bárbara de Queiroz Gadelha' (D), Camila Drumond Muzi² (D), Raphael Mendonça Guimarães ${ }^{3}$ (D) \\ 'Instituto Nacional do Câncer José Alencar Gomes da Silva - Rio de Janeiro (RJ), Brasil. \\ ${ }^{2}$ Instituto de Estudos em Saúde Coletiva, Universidade Federal do Rio de Janeiro (UFRJ) - Rio de Janeiro (RJ), Brasil. \\ ${ }^{3}$ Escola Politécnica de Saúde Joaquim Venâncio, Fundação Osvaldo Cruz (Fiocruz) - Rio de Janeiro (RJ), Brasil.
}

Como citar: Gadelha BQ, Muzi CD, Guimarães RM. Avaliação da satisfação de pacientes oncológicos com o serviço de saú de em um hospital público no Brasil. Cad Saúde Colet, 2020;28(3):353-361. https://doi.org/10.1590/1414$462 \times 202028030175$

\begin{abstract}
Resumo
Introdução: A satisfação dos pacientes é fundamental para melhorar a qualidade dos serviços. Para uniformizar as pesquisas foi criado o EORTC-IN-PATSAT32. Objetivo: O objetivo foi avaliar a satisfação dos pacientes com a internação. Método: Foram entrevistados 322 pacientes, através dos questionários socioeconômico e EORTC-IN-PATSAT32. Os itens múltiplos foram agrupados nas dimensões previstas na versão brasileira do questionário. Foram utilizados testes de hipóteses para avaliar diferenças na avaliação. Resultados: A maioria dos pacientes era do sexo masculino, maiores de 60 anos, baixa escolaridade, casados, em atendimento clínico e sem metástase. Maior nível de satisfação foi com alta hospitalar e maior insatisfação com a estrutura. Houve maior satisfação com a equipe multiprofissional entre os pacientes com menor escolaridade e mais de 40 anos. Os pacientes cirúrgicos e brancos tiveram a maior satisfação com os médicos. Pacientes casados foram os mais satisfeitos com a conduta terapêutica, enquanto homens e idosos foram os mais satisfeitos com a estrutura, e os brancos mais satisfeitos com a alta. Conclusão: Sexo, faixa etária, raça, grau de escolaridade, estado civil e modalidade de tratamento influenciam a satisfação dos pacientes. Recomenda-se o desenvolvimento de estudos em outros hospitais do país para relacionar a satisfação com as características da população brasileira. Palavras-chave: IN-PATSAT32; ambiente hospitalar; inquéritos e questionários; neoplasias; internação hospitalar.
\end{abstract}

\begin{abstract}
Background: Patient satisfaction is fundamental to improve the quality of health services. In order to standardize the surveys, the EORTC-IN-PATSAT32 questionnaire was created. Objective: The objective was to evaluate patients' satisfaction with hospitalization. Method: 322 patients were interviewed through the socioeconomic aspects and EORTC-IN-PATSAT32 questionnaires. The multiple items were grouped in the dimensions provided for in the Brazilian version of the questionnaire. Hypothesis tests were used to evaluate differences in the evaluation. Results: The majority of the patients were male, older than 60 years, low educational level, married, in clinical care and without metastasis. Higher level of satisfaction was with hospital discharge and greater dissatisfaction with the structure. There was greater satisfaction with the multi-professional team among patients with less education and more than 40 years. Surgical and white patients had the greatest satisfaction with physicians. Married patients were the most satisfied with the therapeutic conduct, while men and elderly were the most satisfied with the structure, as long as whites
\end{abstract}

Trabalho realizado no Hospital do Câncer I (HCI-INCA) - Rio de Janeiro (RJ), Brasil.

Correspondência: Bárbara de Queiroz Gadelha. E-mail: bgadelha@inca.gov.br

Fonte de financiamento: nenhuma.

Conflito de interesses: nada a declarar.

Recebido em: Maio 10, 2017. Aprovado em: Ago. 08, 2019
Este é um artigo publicado em sob a licença Creative Commons Attribution, que permite uso, distribuição e reprodução em qualquer meio, sem restrições desde que o trabalho original seja corretamente citado. 
were more satisfied with the discharge. Conclusion: Patient satisfaction is influenced by gender, age, race, education level, marital status and treatment modality. It is recommended the development of studies in other hospitals in the country to relate the satisfaction with the characteristics of the Brazilian population.

Keywords: IN_PATSAT32; hospital environment; surveys, questionnaires; neoplasms; hospital admissions.

\section{INTRODUÇÃO}

O câncer é um problema de saúde pública, com alto impacto socioeconômico, e que envolve tratamento com alto nível de complexidade. No Brasil, estimam-se cerca de 600 mil novos casos no biênio $2016 / 2017^{1}$, e representa a segunda causa de morte no país.

Os tratamentos oncológicos em geral podem incluir procedimentos clínicos e cirúrgicos com eventual necessidade de internação, muitas vezes de forma prolongada. De fato, o ato de hospitalização em si acarreta repercussões muitas vezes negativas ao paciente, que se vê impossibilitado de realizar suas atividades, com ausência do convívio familiar e a necessidade de seguir regras diferentes da sua rotina, ocasionando insatisfação e ansiedade no paciente ${ }^{2}$.

Devido à severidade da doença e os tratamentos sofisticados e suas complicações, a qualidade dos serviços de saúde deve ser considerada e constantemente aferida ${ }^{3}$. Por conta disso, a satisfação dos pacientes com câncer vem sendo reconhecida como essencial na definição de qualidade dos serviços de saúde ${ }^{4}$. Embora os indicadores orientados pela clínica stricto sensu (morbidade e tempo de internação) continuem sendo as métricas de qualidade mais utilizadas, muitas organizações de saúde passaram a considerar os resultados percebidos pelos pacientes (qualidade de vida, satisfação do paciente com os cuidados) como uma medida da qualidade do tratamento ${ }^{5}$. A satisfação do paciente, portanto, tem sido cada vez mais usada como medida de qualidade e desempenho para organizações de saúde. Trata-se de um conceito abstrato e multidimensional, difícil de ser medido e observado ${ }^{6}$.

O EORTC-IN-PATSAT32 foi desenvolvido com o objetivo de prover informações sobre a satisfação dos pacientes oncológicos com os cuidados médicos, cuidados da enfermagem, organização dos cuidados e serviços recebidos no hospital. Esse questionário permite comparar os resultados obtidos em amostras culturalmente diferentes de pacientes oncológicos hospitalizados ${ }^{4}$. Esse instrumento foi submetido ao processo de tradução ${ }^{7}$ e adaptação transcultural para o Brasil ${ }^{8}$.

Deste modo, o questionário EORTC-IN-PATSAT32 foi aplicado neste estudo com o objetivo de avaliar a satisfação dos pacientes oncológicos internados com os serviços médico-hospitalares recebidos, verificando a relação entre a satisfação e as características sociodemográficas.

\section{MÉTODO}

O presente estudo utilizou a versão brasileira traduzida do EORTC-IN-PATSAT32 ${ }^{7}$, e validada no Brasil ${ }^{8}$. Trata-se de estudo transversal, incluídos 322 pacientes internados nas enfermarias clínicas e cirúrgicas do Instituto Nacional de Câncer José Alencar Gomes da Silva (INCA), no Rio de Janeiro, Brasil. Participaram do estudo pacientes com mais de 18 anos, de ambos os sexos.

\section{Coleta de dados}

Os pacientes internados foram abordados e, após concordarem em participar, responderam ao questionário sociodemográfico através de entrevista, seguido do questionário EORTC-IN-PATSAT32. O questionário sociodemográfico e de caracterização do estado clínico incluiu os seguintes aspectos: gênero, idade, raça, grau de escolaridade, estado civil, setor de internação e presença ou ausência de metástase. Já o EORTC-IN-PATSAT32 possui 32 questões relativas a dimensões associadas à satisfação com os cuidados, quais sejam: satisfação com a equipe multiprofissional; satisfação com os médicos; satisfação com a conduta terapêutica; satisfação com a estrutura hospitalar; e satisfação com a alta. A coleta foi realizada entre janeiro e setembro de 2016. 


\section{Análise de dados}

As análises estatísticas foram realizadas utilizado o Excel 2007 e o software SPSS versão 22. As avaliações da confiabilidade das escalas multi-itens do instrumento e da consistência interna foram realizadas através do coeficiente alfa de Cronbach, com o valor mínimo de 0,709.

Os itens múltiplos foram agrupados de acordo com a versão brasileira do questionário EORTC-IN-PATSAT32 ${ }^{8}$ nas escalas: satisfação com equipe multiprofissional (questões 12 a 24 e 32); satisfação com os médicos (questões 2 a 7, 10 e 11); satisfação com a conduta terapêutica (questões 1, 8, 9, 25, 27 e 28); satisfação com a estrutura hospitalar (questões 29 a 31); e satisfação com a alta, com um único item - questão 26 . O resultado bruto de cada item individual foi somado e os resultados referentes às escalas multi-item foram divididos entre o número de itens da escala. Esses resultados obtidos das escalas foram transformados linearmente para obter valores de 0 a 100, em que o valor mais alto indicou o maior nível de satisfação. Para a presente análise, optou-se por categorizar os participantes como"satisfeito"e "insatisfeito". Para isso, procedeu-se à divisão dos respondentes em quartis, e considerou-se o escore do terceiro quartil (q3) como ponto de corte para avaliar a satisfação, para cada dimensão.

A relação com as características sociodemográficas (sexo, raça, grau de escolaridade, estado civil, setor de internação e presença de metástase) foi analisada através do teste de qui-quadrado de Pearson e, quando a frequência foi inferior a 5, utilizou-se o teste exato de Fisher. Os respectivos intervalos de confiança foram calculados a partir da seguinte Fórmula 1:

$$
\hat{\mathrm{p}} \pm 1,96 \mathrm{SE}
$$

Em que $\hat{p}$ representa a proporção calculada para a frequência relativa descrita, e SE representa o erro padrão da proporção amostral, que é calculado a partir da seguinte Fórmula 2:

$$
\sqrt{\frac{\hat{p}(1-\hat{p})}{n}}
$$

Em que $n$ é o tamanho da amostra de estudo.

\section{Considerações éticas}

Os participantes foram submetidos ao Termo de Consentimento Livre e Esclarecido (TCLE), do projeto aprovado pelo Comitê de Ética do INCA. O presente estudo foi devidamente aprovado pelo Comitê de Ética em Pesquisa da instituição, em conformidade com a Portaria $n^{\circ}$ 466/2012, sob o Parecer n 863.339/2014.

\section{RESULTADOS}

Foram entrevistados 322 pacientes. Ao longo do estudo, houve um total de 13 recusas de participação. Houve predominância do sexo masculino (55,8\%), com mais de 60 anos (44,8\%), tendo o intervalo de idade ficado entre 18 e 98 anos. Observou-se também que a maioria das pessoas se declararam casadas (59,5\%), com a escolaridade correspondente ao ensino fundamental completo ou incompleto (45,7\%), apenas $16,5 \%$ da amostra possuía nível superior. Com relação à cor de pele, os grupos de brancos e pretos/pardos/amarelos apresentaram frequência muito próxima ( $50,5 \%$ vs. $49,5 \%$, respectivamente). Houve maior frequência de pacientes clínicos $(63,7 \%)$ e com ausência de metástase (68,9\%) (Tabela 1).

Na Tabela 2, evidenciou-se o alto índice de satisfação em todas as dimensões do questionário e em geral. Destaca-se a menor satisfação com a estrutura hospitalar, e a maior com a alta, seguida pela satisfação com o atendimento da equipe médica.

Quando analisadas as dimensões de satisfação de acordo com as categorias das variáveis selecionadas (Tabela 3), com relação à equipe, há uma diferença estatisticamente significativa para a faixa etária e escolaridade, com maior grau de satisfação entre os pacientes mais velhos e entre menos escolarizados alfabetizados. Para a relação com os médicos, apresentaram maior satisfação os pacientes dos setores clínicos. Com relação à conduta terapêutica, os pacientes casados tiveram maior satisfação. A estrutura hospitalar pareceu mais adequada aos 
Tabela 1. Características demográficas e de natureza clínica da população de estudo $(n=322)$, Rio de Janeiro - RJ, 2016.

\begin{tabular}{|c|c|c|}
\hline Dados socioeconômicos & $\mathbf{n}$ & $\%$ \\
\hline \multicolumn{3}{|l|}{ Sexo } \\
\hline Masculino & 180 & 55,8 \\
\hline Feminino & 142 & 44,2 \\
\hline \multicolumn{3}{|l|}{ Faixa etária } \\
\hline 18 a 39 anos & 50 & 15,5 \\
\hline 40 a 59 anos & 127 & 39,6 \\
\hline 60 anos ou mais & 145 & 44,8 \\
\hline \multicolumn{3}{|l|}{ Raça } \\
\hline Branco & 161 & 50 \\
\hline Preto ou pardo & 159 & 49,4 \\
\hline Amarelo & 2 & 0,6 \\
\hline \multicolumn{3}{|l|}{ Escolaridade } \\
\hline Analfabeto & 18 & 5,5 \\
\hline Fundamental & 147 & 45,7 \\
\hline Médio & 104 & 32,3 \\
\hline Superior & 53 & 16,5 \\
\hline \multicolumn{3}{|l|}{ Estado civil } \\
\hline Solteiro & 66 & 20,4 \\
\hline Casado & 191 & 59,5 \\
\hline Viúvo ou separado & 65 & 20,1 \\
\hline \multicolumn{3}{|l|}{ Setor de internação } \\
\hline Clínico & 205 & 63,7 \\
\hline Cirúrgico & 117 & 36,3 \\
\hline \multicolumn{3}{|l|}{ Metástase } \\
\hline Sim & 90 & 28,0 \\
\hline Não & 222 & 68,9 \\
\hline Ignorado & 10 & 3,1 \\
\hline Total & 322 & 100 \\
\hline
\end{tabular}

Tabela 2. Avaliação da satisfação dos pacientes com cada dimensão do questionário EORTC-IN-PATSAT32 $(\mathrm{n}=322)$, Rio de Janeiro - RJ, 2016.

\begin{tabular}{lccccc} 
& \multirow{2}{*}{ Dimensões } & \multicolumn{2}{c}{ Satisfeito } & & \multicolumn{2}{c}{ Insatisfeito } \\
\cline { 2 - 5 } \cline { 5 - 6 } & $\mathbf{n}$ & $\%$ & & $\mathbf{n}$ & $\%$ \\
\hline Equipe multiprofissional & 294 & 89,6 & & 34 & 10,4 \\
\hline Médicos & 307 & 93,6 & & 21 & 6,4 \\
\hline Conduta terapêutica & 298 & 90,9 & & 30 & 9,1 \\
\hline Estrutura hospitalar & 235 & 71,6 & & 93 & 28,4 \\
\hline Alta & 310 & 94,5 & & 18 & 5,5 \\
\hline Geral & 302 & 92,1 & 26 & 7,9 \\
\hline
\end{tabular}




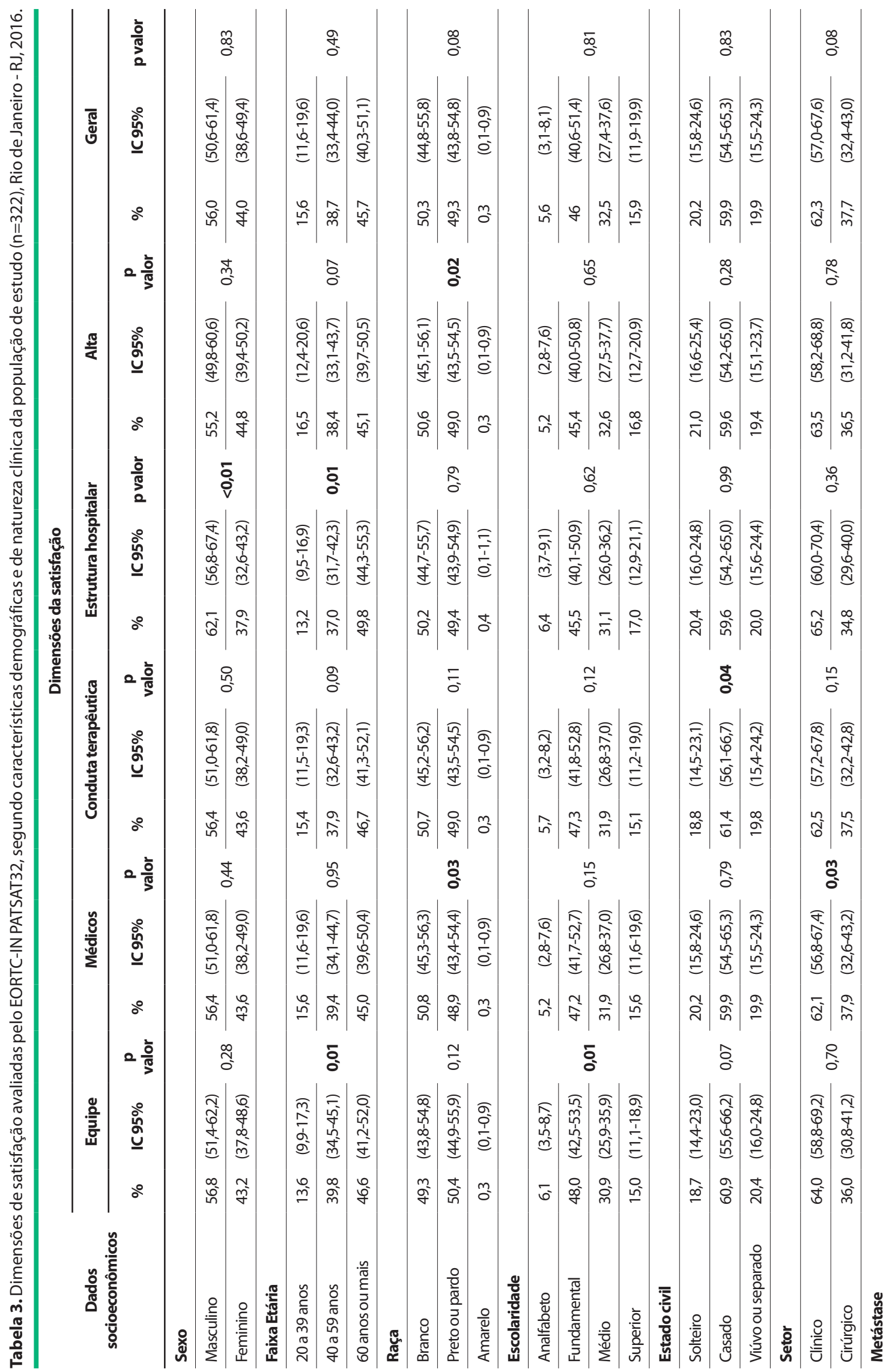


pacientes do sexo masculino e mais velhos, e a satisfação com os procedimentos de alta não apresentou diferença para nenhuma característica social, demográfica ou clínica. Finalmente, em uma avaliação geral, não foram identificadas associações estatisticamente significativas, evidenciando que eventuais diferenças são pontuais.

\section{DISCUSSÃO}

As abordagens para o enfrentamento do câncer são diversas, e seu controle inclui políticas públicas que considerem aspectos clínicos e sociais ${ }^{1}$. Nesse contexto, é notório que a capacidade dos profissionais e cuidadores para reconhecer e entender as necessidades dos pacientes é um dos principais determinantes para a sua satisfação com o cuidado oferecido ${ }^{10}$. O questionário EORTC-IN-PATSAT32, desenvolvido pela EORTC, já foi adaptado e validado para diversas culturas ao redor do mundo e vem sendo aplicado para avaliar a satisfação dos

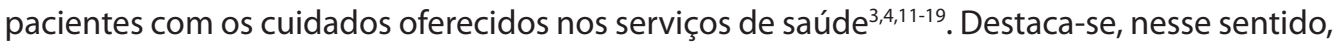
a relação entre a satisfação e as variáveis socioeconômicas, que podem ser definidoras de diferentes abordagens aos pacientes, de acordo com necessidades que não estão diretamente relacionadas à prática do cuidado, e sim à questão do acesso, da disponibilidade em estar internado com acompanhantes, do nível de entendimento sobre as orientações, entre outros. Dessa forma, é importante reconhecer que características ligadas ao contexto de vida são igualmente importantes para o tratamento. Essa argumentação foi inserida na discussão.

Esses estudos são fundamentais para identificar as necessidades dos pacientes e destacar quais áreas do serviço de saúde podem ser melhoradas. É importante citar que estudos anteriores apontaram, ainda, que não há diferença significativa entre a satisfação dos pacientes e o tempo de internação ${ }^{20}$. Face a isso, essa característica da internação não foi verificada.

Há, contudo, que se perceber diferenças entre os estudos, que particularizam o Brasil nesse contexto. $O$ perfil dos pacientes participantes do estudo apresentou maior frequência de idosos e com baixo nível de escolaridade, ao contrário da amostra utilizada por Bredart et al. ${ }^{4}$.

A satisfação com a equipe multidisciplinar foi maior entre os pacientes com baixa escolaridade em relação aos pacientes com ensino médio e superior. Anteriormente, também foi verificada maior satisfação entre os pacientes com menor grau de escolaridade ${ }^{10,21}$ e menor nível de satisfação com as habilidades dos médicos em pacientes com nível universitário ${ }^{16}$. Além disso, observou-se diferença significativa entre homens e mulheres com relação à estrutura do hospital ${ }^{16}$. Ainda, foi constatado que não há diferença significativa entre a satisfação e as enfermidades ou modalidades de tratamento ${ }^{22}$.

Ressalta-se, aqui, o perfil dos serviços em que o estudo foi conduzido (cirurgia abdominal, cirurgia de cabeça e pescoço, hematologia, oncologia clínica, cirurgia torácica e urologia). De fato, estudos anteriores envolvendo pacientes cirúrgicos apontaram diferenças entre os pacientes que tiveram complicações pós-operatórias e os que não tiveram, com relação à estrutura hospitalar ${ }^{23}$. Esse aspecto não foi verificado. Entretanto, o setor de internação - clínico ou cirúrgico - apresentou diferenças entre os níveis de satisfação com relação ao atendimento dos médicos.

A maior satisfação registrada foi com a alta hospitalar, o que pode ser reflexo da satisfação em geral com a qualidade do serviço oferecido. Outra questão a ser considerada, a esse respeito, é a expectativa gerada pelos pacientes ao redor da alta, uma vez que desejam o momento em que poderão retornar para o tratamento ambulatorial nas suas residências.

A estrutura hospitalar também foi alvo do menor nível de satisfação encontrado $(71,6 \%)$. Esse resultado concorda com as principais reclamações realizadas pelos pacientes no ato da entrevista, onde muitos reclamaram da ausência de botões para chamado de emergência no leito (ausentes ou danificados), da higienização dos banheiros e de ausência de água quente em determinados períodos. Além disso, muitos pacientes comentaram as dificuldades encontradas para acessar o hospital, mas não as atribuíram à administração do hospital, e sim a um problema de mobilidade urbana. Outras reclamações foram registradas, como a heterogeneidade da equipe de enfermagem, refletindo na segunda pior avaliação da satisfação do estudo para a equipe multiprofissional. Ainda, os resultados do nosso estudo 
parecem mostrar que é essencial que todos os membros da equipe de saúde estejam cientes dos serviços de saúde e cuidados necessários para pacientes com câncer, a fim de promover a satisfação do paciente. Isto é observável quando se tem diferenças no nível de satisfação entre as dimensões, ou entre questões relativas ao cuidado que são privativas de um ou outro profissional da equipe de saúde. Essa evidência é relativamente consensual na literatura, com destaque para a maior satisfação quando há suporte para questões médicas e sociais ${ }^{24}$; abordagem a partir da dignidade no cuidado ${ }^{25,26}$; a passagem de plantões na equipe realizada à beira do leito ${ }^{27}$; e comunicação, cuidado personalizado, foco centrado na família, consistência na equipe multiprofissional e ambiente de apoio ${ }^{28,29}$.

O presente estudo apontou, dessa forma, que certos fatores relacionados ao paciente, às instituições e à cultura possuem diferenças com significância estatística com a percepção da qualidade do tratamento do câncer. Esse resultado corrobora avaliação de estudo semelhante realizado em países asiáticos e europeus ${ }^{30,31}$. É importante, contudo, ressaltar que há marcadas diferenças, nesses aspectos, entre Brasil e esses países, principalmente considerando a natureza do sistema de saúde (no caso brasileiro, universal e gratuito), as questões culturais (que definem, no limite, o enfrentamento da doença). Uma recente revisão sistemática ${ }^{32}$ destacou a validade desse instrumento em distintos contextos. Contudo, o Brasil não esteve incluído neste estudo, possivelmente pelo fato de o estudo de validação, assim como o estudo no Marrocos ${ }^{33}$, terem sido publicados posteriormente ao protocolo daquela revisão.

Ainda, o contexto sociocultural da população estudada a torna única, pois, apesar do enorme aprimoramento e desenvolvimento no campo da saúde pública, em especial da atenção oncológica, as redes de serviços ainda são incipientes e pouco reconhecidas pelos pacientes e profissionais da área clínica. Recomenda-se, nesse sentido, que haja desdobramento do presente estudo, com uma amostra maior, mais robusta e diversificada, que dê conta de capturar diferenças para características mais específicas, como por exemplo o fato de os pacientes terem suporte familiar e social, ou para o fato de se encontrarem em protocolo curativo ou paliativo. Com isso, garante-se a representação ideal de regiões e culturas, e de sistemas e estruturas de cuidados em saúde. Assim, será possível esclarecer adequadamente a relação entre paciente, fatores clínicos e contextuais e a satisfação do paciente.

Alguns pontos merecem destaque. Embora a escala final atribua um escore por cada paciente, optou-se por categorizar o grupo, na tentativa de classificar cada sujeito como satisfeito ou não com o serviço. Optou-se por utilizar a divisão por quartis, e considerar aquele de maior escore como satisfeito. Admite-se que essa escolha pode resultar em um erro de classificação, pois o ponto de corte pode aumentar ou diminuir tanto a sensibilidade quanto a especificidade do teste. No entanto, como não há valor de cut-off estabelecido na literatura, optou-se por fazer a classificação utilizando separatrizes. Na medida em que a amostra não permitia a divisão em grupos menores, pareceu adequado o uso de quartis. É importante ressaltar que essa estratégia é útil, principalmente, pela tentativa de trazer uma opção à aplicação do instrumento, para que se possa demonstrar a factibilidade de sua utilização na rotina dos serviços.

A satisfação dos pacientes com o atendimento na internação hospitalar está relacionada com o sexo, faixa etária, raça, grau de escolaridade, estado civil e modalidade de tratamento (clínico ou cirúrgico). Os pacientes com menor escolaridade e mais de 40 anos são os mais satisfeitos com o atendimento da equipe multiprofissional. Os médicos obtiveram maiores níveis de satisfação entre pacientes brancos e em tratamento cirúrgico. Os pacientes casados foram os mais satisfeitos com a conduta hospitalar, enquanto os homens idosos se destacaram como mais satisfeitos com a estrutura hospitalar, e os brancos, com a alta.

O presente estudo fornece uma visão sobre a prestação de serviços de saúde em oncologia. Contudo, é preciso reconhecer que, a despeito de se tratar de uma instituição pública, é um hospital de alta complexidade, especializado em oncologia. Dessa forma, é preciso considerar que hospitais que sejam gerais, ou que sejam públicos, mas em outra esfera de governo (estadual e municipal), tenham diferenças importantes de avaliação. Destarte, recomenda-se contextualizar a instituição em avaliação, e não ter a pretensão de generalizações. Por isso, 
reconhece-se como potencialidade deste estudo a tentativa de aplicação desse instrumento, a fim de se observar se é uma ação possível de ser introduzida na rotina dos serviços.

\section{REFERÊNCIAS}

1. Instituto Nacional de Câncer José Alencar Gomes da Silva. Coordenação de Prevenção e Vigilância. Estimativa 2018: incidência de câncer no Brasil [Internet]. 2018 [citado em 2007 Maio 10]. Disponível em: http://www.inca.gov.br/wcm/dncc/2017/dados-apresentados.pdf

2. Oliveira AML, Guirardello EB. Satisfação do paciente com os cuidados de enfermagem: comparação ente dois hospitais. Rev Esc Enferm USP. 2006;40(1):71-7. http://dx.doi.org/10.1590/S0080-62342006000100010. PMid:16719130.

3. Pishkuhi MA, Salmaniyan S, Nedjat S, Zendedel K, Lari MA. Psychometric properties of the persian version of satisfaction with care EORTC-IN-PATSAT32 questionnaire among Iranian cancer patients. Asian Pac J Cancer Prev. 2014;15(23):10121-8. http://dx.doi.org/10.7314/APJCP.2014.15.23.10121. PMid:25556436.

4. Brédart A, Coens C, Aaronson N, Chie W-C, Efficace F, Conroy T, et al. and the EORTC Quality of Life Group and EORTC Quality of Life Unit. Determinants of patient satisfaction in oncology settings from European and Asian countries: preliminary results based on the EORTC IN-PATSAT32 questionnaire. Eur J Cancer. 2007;43(2):323-30. http://dx.doi.org/10.1016/j.ejca.2006.10.016. PMid:17156997.

5. Papastavrou E, Andreou P, Tsangari H, Merkouris A. Linking patient satisfaction with nursing care: the case of care rationing - a correlational study. BMC Nurs. 2014;13(1):26. http://dx.doi.org/10.1186/14726955-13-26. PMid:25285040.

6. Shiva A, Haden SC, Brooks J. Psychiatric civil and forensic inpatient satisfaction with care: the impact of provider and recipient characteristics. Soc Psychiatry Psychiatr Epidemiol. 2009;44(11):979-87. http:// dx.doi.org/10.1007/s00127-009-0019-3. PMid:19271090.

7. Belmiro AA, Carvalho MS, Viana ÁFV, Rocha LF, Jomar RT, Muzi CD, et al. Equivalência conceitual, de itens, semântica e operacional da versão brasileira do EORTC IN-PATSAT32. Rev Augustus. 2018 dez;23(46):6981. http://dx.doi.org/10.15202/1981896.2018v23n46p69.

8. Gadelha BQ, Muzi CD, Guimarães RM. Transcultural adaptation of the user satisfaction scale to the health service: Brazilian version of the EORTC IN-PATSAT32 questionnaire. Clin TransI Oncol. 2018 jun;20(6):74552. http://dx.doi.org/10.1007/s12094-017-1780-5. PMid:29086251.

9. Rowland D, Arkkelin D, Crisler L. Computer-based data analysis: using SPSS in the Social and Behavioral Sciences. Chicago: Wardsworth Pub Co; 1991.

10. Leo F, Radice D, Didier F, Carvalho FM, Spacca E, Madini M, et al. Does a personalized approach improve patient satisfaction in thoracic oncology? Am J Manag Care. 2009;15(6):361-7. PMid:19514802.

11. Poinsot R, Altmeyer A, Conroy T, Savignoni A, Asselain B, Léonard I, et al. Validation multicentrique d'un questionnaire de satisfaction des soins lors d'un traitement de chimiothérapie ou radiothérapie ambulatoire. Bull Cancer. 2006;93(3):315-27. PMid:16567319.

12. Jayasekara H, Rajapaksa L, Bredart A. Psychometric evaluation of the European Organization for Research and Treatment of Cancer in-patient satisfaction with care questionnaire ('Sinhala' version) for use in a South-Asian setting. Int J Qual Health Care. 2008;20(3):221-6. http://dx.doi.org/10.1093/intqhc/mzn006. PMid:18339668.

13. Arraras Jl, Vera R, Martinez M, Hernandez B, Lainez N, Rico M, et al. The EORTC cancer in-patient satisfaction with care questionnaire: EORTC IN-PATSAT32 Validation study for Spanish patients. Clin TransI Oncol. 2009;11(4):237-42. http://dx.doi.org/10.1007/s12094-009-0346-6. PMid:19380301.

14. Arora V, Philp S, Nattress K, Pather S, Dalrymple C, Atkinson K, et al. Patient satisfaction with inpatient care provided by the Sydney Gynecological Oncology Group. Patient Relat Outcome Meas. 2010;1:179-84. http://dx.doi.org/10.2147/PROM.S13897. PMid:22915963.

15. Hjörleifsdóttir E, Hallberg IR, Gunnarsdóttir E. Satisfaction with care in oncology outpatient clinics: psychometric characteristics of the Icelandic EORTC IN-PATSAT32 version. J Clin Nurs. 2010;19(13-14):178494. http://dx.doi.org/10.1111/j.1365-2702.2009.03095.x. PMid:20920006.

16. Obtel M, El Achhab Y, Bendahhou K, Benider A, Errihani H, Zidouh A, et al. Measuring patient satisfaction in Moroccan oncology institutions EORTC IN-PATSAT32 and satisfaction in Moroccan patient with cancer. J Afr Cancer. 2012;4(1):9-17. http://dx.doi.org/10.1007/s12558-011-0185-6.

17. Scarpa M, Di Cristofaro L, Cortinovis M, Pinto E, Massa M, Alfieri R, et al. Minimally invasive surgery for colorectal cancer: quality of life and satisfaction with care in elderly patients. Surg Endosc. 2013;27(8):291120. http://dx.doi.org/10.1007/s00464-013-2854-2. PMid:23468328. 
18. Zhang J, Xie S, Liu J, Sun W, Guo H, Hu Y, et al. Validation of EORTC IN-PATSAT32 for Chinese patients with gastrointestinal cancer. Patient Prefer Adherence. 2014;8(8):1285-92. PMid:25258522.

19. Brinkman-Stoppelenburg A, Polinder S, Vergouwe Y, Van Der Heide A. Palliative care consultation services in hospitals in the Netherlands: the design of the COMPASS study. BMC Palliat Care. 2015;14(1):68-74 http://dx.doi.org/10.1186/s12904-015-0069-0. PMid:26626877.

20. Pompili C, Tiberi M, Salati M, Refai M, Xiume F, Brunelli A. Patient satisfaction with health-care professionals and structure is not affected by longer hospital stay and complications after lung resection: a casematched analysis. Interact Cardiovasc Thorac Surg. 2015;20(2):236-41. http://dx.doi.org/10.1093/icvts/ ivu371. PMid:25376724.

21. Mendonça KMPP, Guerra RO, Diógenes TPM, Lima TMFS. Influência das características sócio-demográficas na satisfação do paciente com o tratamento fisioterapêutico. Fisioter Mov. 2006;19:83-9.

22. Balderas-Peña LM, Sat-Muñoz D, Contreras-Hernández I, Solano-Murillo P, Hernández-Chávez GA, MariscalRamírez I, et al. Evaluation of patient satisfaction with the quality of health care received within the EORTC IN-PATSAT32 trial by patients with breast and colorectal cancer, and non-Hodgkin lymphoma at different stages. Correlation with sociodemographic characteristics, comorbidities and other procedural variables at the Mexican Institute of Social Security. Value Health. 2011;14(5 Suppl 1):S96-9. PMid:21839910.

23. Barleísi F, Boyer L, Doddoli C, Antoniotti S, Thomas P, Auquier P. The place of patient satisfaction in quality assessment of lung câncer thoracic surgery. Chest. 2005;128(5):3475-81. http://dx.doi.org/10.1378/ chest.128.5.3475. PMid:16304302.

24. Zimmermann C, Swami N, Krzyzanowska M, Hannon B, Leighl N, Oza A, et al. Early palliative care for patients with advanced cancer: a cluster-randomised controlled trial. Lancet. 2014;383(9930):1721-30. http://dx.doi.org/10.1016/S0140-6736(13)62416-2. PMid:24559581.

25. Chand P, Gabriel T, Wallace CL, Nelson CM. Inpatient palliative care consultation: Describing patient satisfaction. Perm J. 2013;17(1):53-5. http://dx.doi.org/10.7812/TPP/12-092. PMid:23596370.

26. Aboshaiqah A, Al-Saedi TS, Abu-Al-Ruyhaylah MM, Aloufi AA, Alharbi MO, Alharbi SS, et al. Quality of life and satisfaction with care among palliative cancer patients in Saudi Arabia. Palliat Support Care. 2016;14(6):621-7. http://dx.doi.org/10.1017/S1478951516000432. PMid:27323905.

27. Kullberg A, Sharp L, Johansson H, Brandberg Y, Bergenmar M. Patient satisfaction after implementation of person-centred handover in oncological inpatient care - A cross-sectional study. PLoS One. 2017 abr;12(4):e0175397. http://dx.doi.org/10.1371/journal.pone.0175397. PMid:28384314.

28. Wentlandt K, Seccareccia D, Kevork N, Workentin K, Blacker S, Grossman D, et al. Quality of care and satisfaction with care on palliative care units. J Pain Symptom Manage. 2016;51(2):184-92. http://dx.doi. org/10.1016/j.jpainsymman.2015.10.006. PMid:26598036.

29. Aboshaiqah A, Al-Saedi TS, Abu-Al-Ruyhaylah MM, Aloufi AA, Alharbi MO, Alharbi SS, et al. Quality of life and satisfaction with care among palliative cancer patients in Saudi Arabia. Palliat Support Care. 2016;14(6):621-7. http://dx.doi.org/10.1017/S1478951516000432. PMid:27323905.

30. Brédart A, Coens C, Aaronson N, Chie WC, Efficace F, Conroy T, et al. Determinants of patient satisfaction in oncology settings from European and Asian countries: preliminary results based on the EORTC INPATSAT32 questionnaire. Eur J Cancer. 2007;43(2):323-30. http://dx.doi.org/10.1016/j.ejca.2006.10.016. PMid:17156997.

31. Brédart A, Bottomley A, Blazeby JM, Conroy T, Coens C, D'haese S, et al. An international prospective study of the EORTC cancer in-patient satisfaction with care measure (EORTC IN-PATSAT32). Eur J Cancer. 2005;41(14):2120-31. http://dx.doi.org/10.1016/j.ejca.2005.04.041. PMid:16182120.

32. Neijenhuijs KI, Jansen F, Aaronson NK, Brédart A, Groenvold M, Holzner B, et al. A systematic review of the measurement properties of the European Organisation for Research and Treatment of Cancer In-patient Satisfaction with Care Questionnaire, the EORTC IN-PATSAT32. Support Care Cancer. 2018;26(8):2551-60. http://dx.doi.org/10.1007/s00520-018-4243-9. PMid:29732482.

33. Obtel M, Serhier Z, Bendahhou K, Bennani M, Zidouh A, Benider A, et al. Validation of EORTC IN-PATSAT 32 in Morocco: Methods and Processes. Asian Pac J Cancer Prev. 2017;18(5):1403-9. PMid:28612594. 